\section{EI BID y las políticas de Ciencia, Tecnología e Innovación en Argentina (1990-2015)}

Resumen: Este trabajo da cuenta de cómo se estructuró, entre 1990 y 2015, la política de ciencia, tecnología e innovación en Argentina, y el papel que cumplió en ello el Banco Interamericano de Desarrollo (BID), a través de los créditos otorgados en ese período. En este marco se analiza la creación de la Agencia Nacional de Promoción Científica y Tecnológica (ANPCyT), el rol significativo que tuvo el BID en su consolidación y cómo mediante sucesivos acuerdos con este organismo, la ANPCyT fue introduciendo cambios que generaron una oferta diversificada de instrumentos de financiamiento. Se discute el papel que sigue cumpliendo el BID en la financiación de los fondos para investigación y desarrollo en el país y se reflexiona sobre los alcances y limitaciones que presentan estas políticas.

Palabras clave: Políticas CTI en Argentina, créditos del BID, ANPCyT.

The IDB and the Science, Technology and Innovation policies in Argentina (1990-2015)

Abstract: This paper shows how the science, technology and innovation policy in Argentina was structured between 1990 and 2015, focusing on the role played by the Inter-American Development Bank (IDB) through the loans granted in that period. In this framework, the creation of the National Agency for Scientific and Technological Promotion (ANPCyT) is discussed in relation with the significant role of the IDB in its consolidation and how -through successive agreements with this organization-, the ANPCyT introduced changes that generated a diversified supply of financing instruments. The role that the IDB continues to play in financing research and development funds in the country is discussed and the scope and limitations of these policies are reflected.

Keywords: CTI policies in Argentina, IDB credits, ANPCyT.

O BID e as políticas de ciência, tecnologia e inovação na Argentina (1990-2015)

Resumo: Este artigo mostra como a política de ciência, tecnologia e inovação na Argentina foi estruturada entre 1990 e 2015 e o papel desempenhado pelo Banco Interamericano de Desenvolvimento (BID) através dos empréstimos concedidos naquele período. . Nesse contexto, a criação da Agência Nacional de Promoção Científica e Tecnológica (ANPC\&T); o papel significativo do BID em sua consolidação e como, através de sucessivos acordos com essa organização, a ANPC\&T estava introduzindo mudanças que gerariam uma oferta diversificada de instrumentos de financiamento; e, por fim, o papel que o BID continua desempenhando no financiamento de fundos de pesquisa e desenvolvimento no país são assuntos discutidos juntamente ao escopo e as limitações dessas políticas.

Palavras chave: Políticas de CTI na Argentina, créditos do BID, ANPC\&T.

\section{Ciencia Tecnología y Política \\ Año 2 N 3 jul- dic 2019}

Francisco J. Aristimuño

Doctor en Economía

CITECDE-UNRN y CONICET faristimuno@unrn.edu.ar

Manuel J. Lugones Licenciado en Sociología

CITECDE-UNRN

mlugones@unrn.edu.ar

Año $2 \mathrm{~N}^{\circ} 3 \mathrm{Jul}$ - dic 2019 Fecha de recibido: 26/06/2019 Fecha de aprobado: 07/10/2019 https://doi.org/10.24215/26183188e031 https://revistas.unlp.edu.ar/CTyP ISSN 2618-3188

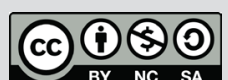

Esta obra está bajo licencia Creative Commons Atribución-NoComercial-Compartirlgual 4.0 Internacional http://creativecommons.org/licenses/bync-sa/4.O/deed.es_AR 
| Ciencia, Tecnología y Política | Año 2 | N³ | Julio-Diciembre 2019 | ISSN 2618-3188 | www.revistas.un|p.edu.ar/CTyP |

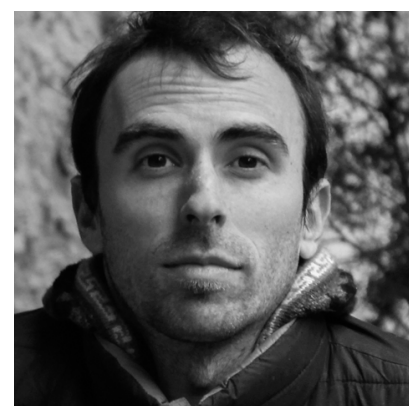

Francisco J. Aristimuño

Doctor en Economía CITECDE-UNRN y CONICET faristimuno@unrn.edu.ar

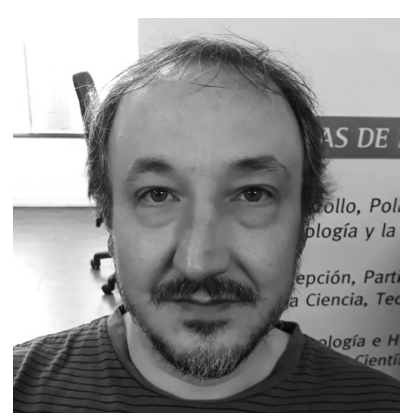

Manuel J. Lugones

\section{El BID y las políticas de Ciencia, Tecnología e Innovación en Argentina (1990-2015)}

Resumen: Este trabajo da cuenta de cómo se estructuró, entre 1990 y 2015, la política de ciencia, tecnología e innovación en Argentina, y el papel que cumplió en ello el Banco Interamericano de Desarrollo (BID), a través de los créditos otorgados en ese período. En este marco se analiza la creación de la Agencia Nacional de Promoción Científica y Tecnológica (ANPCyT), el rol significativo que tuvo el BID en su consolidación y cómo mediante sucesivos acuerdos con este organismo, la ANPCyT fue introduciendo cambios que generaron una oferta diversificada de instrumentos de financiamiento. Se discute el papel que sigue cumpliendo el BID en la financiación de los fondos para investigación y desarrollo en el país y se reflexiona sobre los alcances y limitaciones que presentan estas políticas.

\section{Introducción}

Este trabajo se propone analizar cómo se estructuró, entre 1990-2015, la política de ciencia, tecnología e innovación (CTI) en Argentina a partir de los créditos otorgados por el Banco Interamericano de Desarrollo (BID). La relación de Argentina con el BID en CTI viene de larga data. Desde el comienzo de sus actividades en la década del '60, el BID ha financiado la CTI a lo largo de toda América Latina, pero Argentina ha sido quien más apoyo recibió en ese sector. A partir de la década del '90 el flujo de créditos se incrementó significativamente y se direccionó a una única institución del complejo de CTI nacional, la Agencia Nacional de Promoción Científica y Tecnológica (ANPCyT). Esta institución fue creada en esa misma década y, a pesar de la relevancia que ha logrado en el complejo de instituciones argentinas, sigue dependiendo de los créditos del BID aún en la actualidad.

En el período analizado se llevaron a cabo profundas transformaciones en la estructura de gobernanza del complejo nacional de CTI como consecuencia de la introducción de nuevos mecanismos de intervención estatal. El BID acompañó estas transformaciones a través de sucesivos créditos 
para el sector. Pero ¿hasta qué punto estos créditos influyeron en el desenvolvimiento de las políticas CTI argentinas? ¿Cuál es el peso real del BID en el financiamiento de las actividades de CTI de Argentina? ¿EI BID siempre fue un facilitador de las transformaciones o se opuso a alguna de ellas? A continuación, se recorren 25 años de la relación del BID con Argentina en temas de CTI con el fin de arrojar un poco de luz a estas cuestiones.

En lo que sigue primero reflexionaremos brevemente sobre el viraje de paradigma que aconteció en los 90s. y que el BID acompañó. Luego analizaremos el peso que tuvo el BID en el financiamiento de la CTI argentina en el período estudiado. Posteriormente pasaremos a considerar en más detalle qué financió el BID en cada una de sus operatorias. Finalmente haremos algunas reflexiones sobre el rol del BID en el desarrollo de las políticas CTI de Argentina.

\section{Nuevo enfoque para las políticas de CTI en los años '90s}

Las críticas al modelo de industrialización por sustitución de importaciones (ISI), a partir de mediados de la década del setenta, llevaron al cuestionamiento de los mecanismos de intervención estatal. De este modo, en la década del '90 se llevaron adelante un conjunto de reformas que implicó el desmantelamiento parcial de las políticas de CTI de índole sectorial características del modelo sustitutivo ${ }^{1}$, y su reemplazo por un enfoque de subsidio horizontal a la demanda, lo que implicó una drástica reducción del papel del Estado en la planificación del desarrollo (Sztulwark, 2010). En otros términos, se reemplazó una política de CTI caracterizada por la oferta pública de conocimiento y capacidades de desarrollo tecnológico e innovativo, por el finan- ciamiento directo, por ejemplo mediante subsidios, de la demanda tecnológica de las empresas. En este sentido, se buscó trasladar la responsabilidad de planificar las actividades de CTI, del Estado a las empresas demandantes de tecnologías y servicios tecnológicos (Aristimuño, 2019).

De esta forma, en Argentina, a principios de la última década del siglo pasado, las políticas de CTI se reformularon definiendo a las empresas como los agentes centrales del proceso de innovación. Esto se hizo por medio de la sanción, en septiembre de 1990, de la Ley 23.877 de Promoción y Fomento de la Innovación Tecnológica. Esta ley estableció un marco normativo para la promoción de la innovación dentro de las empresas, mediante la creación de instrumentos financieros (subsidios y créditos concesionales) y no financieros (incentivo fiscal) que debían ser otorgados mediante mecanismos competitivos que permitieran detectar las oportunidades tecnológicas por criterios de excelencia. El elemento novedoso de estos instrumentos fue constituir a las empresas en beneficiarias directas de los fondos públicos de promoción. Hasta la promulgación de la Ley las unidades beneficiarias de los instrumentos habían sido siempre los organismos públicos descentralizados (por ejemplo: Instituto Nacional de Tecnología Industrial -INTI-, Instituto Nacional de Tecnología Agropecuaria -INTA-, Comisión Nacional de Energía Atómica -CNEA-), entre cuyas funciones estaban la prestación de servicios al sector privado. En este esquema, si bien los beneficiarios podían ser actores privados, la ejecución y, por ende, los recursos físicos y humanos necesarios para llevar a cabo la actividad eran públicos.

Otro rasgo disruptivo de la Ley 23.877 fue la creación de la figura de Unidades de Vinculación Tecnológica (UVTs). Las UVTs son entes no estatales

\footnotetext{
${ }^{1}$ Hacemos referencia al conjunto de políticas que desde mediados de la década del cincuenta del siglo pasado se caracterizaron por generar instituciones de I+D y empresas públicas en sectores considerados estratégicos para impulsar el proceso de industrialización.
} 
constituidos para la identificación, selección y formulación de proyectos de I+D, transmisión de tecnología y asistencia técnica. En otros términos, a través de las mismas, se buscó resolver la demanda de una mayor vinculación y transferencia de tecnología entre las instituciones públicas de CTI y el sector privado. Por otro lado, además de buscar una mayor vinculación del complejo con el sector productivo, se perseguía disminuir el financiamiento público del sector a través del acceso a fondos privados. Y, por último, crear un mercado de consultoría y asistencia técnica.

Los nuevos instrumentos también se proponían incrementar la inversión en $1+D+i$ respecto del $\mathrm{PBI}$, sobre la base de una creciente participación del sector privado. En consecuencia, en su diseño se introdujeron mecanismos para promover la inversión privada (adicionalidad). De este modo, el sector público no financiaba el 100\% del valor de los proyectos con créditos, subsidios o incentivos fiscales, sino sólo una porción, de modo que, para acceder a los recursos públicos el actor privado debía aportar una parte del valor del proyecto con recursos propios.

Estos cambios pretendían además traspasar la responsabilidad de determinar el uso de los recursos públicos volcados al complejo de CTI, del sector público al privado a partir de un modelo de política tecnológica horizontal (Teubal, 1990). De acuerdo a Anlló \& Peirano (2005), tres son los factores que determinaron la elección de un modelo de política horizontal: 1- promover conductas innovativas en el conjunto del tejido productivo, 2- tener menores requerimientos de capacidades de gestión estatal para su implementación respecto de los mecanismos selectivos y sectoriales y 3 - evitar comportamientos de búsqueda de captura de rentas que genera el exceso de intervención estatal, es decir, corregir las denominadas fallas de gobierno.

No obstante la profundidad de las transformaciones operadas en los 90's, subsistió un conjunto heterogéneo de acciones e instituciones que responden a diferentes visiones de intervención. En otras palabras, la consolidación de este nuevo esquema de incentivo al cambio tecnológico no llevó al reemplazo de las instituciones creadas a fines de la década del '50 en el marco de la estrategia ISI, por nuevas instituciones que se enmarcaran en la nueva visión horizontal. Lo que ocurrió es que se sumaron nuevas instituciones al complejo de instituciones pre-existentes, haciendo escasos esfuerzos por lograr un marco de intervención coherente en la temática. Lavarello \& Saravia (2015) describen esta situación en términos de la existencia de diferentes "capas geológicas" en la estructura institucional de CTI del Estado argentino. En consecuencia, a partir de los 90's, conviven en el Estado una diversidad de instrumentos e instituciones creados en distintos momentos que remiten a diferentes diagnósticos, objetivos e ideas subyacentes sobre la intervención del Estado en los procesos de crecimiento y desarrollo por la vía de la CTI (Aristimuño, 2019; Lavarello \& Sarabia, 2015).

A partir de 2003, se registró un fuerte incremento en el presupuesto destinado al sector, pero se mantuvo la misma estructura institucional que se configuró hacia finales de los 90's². En este período las políticas de CTI tendieron a impulsar un conjunto de instrumentos de carácter sectorial y de promoción de tecnologías de propósito general (TPG) -o "portadoras de futuro"para la solución de demandas tecnológicas en diferentes "áreas o núcleos socio-productivos" (Hurtado, Lugones, \& Surtayeva, 2017). Esto im-

2 Con la notable excepción de la elevación de la Secretaría de Ciencia y Tecnología al rango de ministerio en 2007. 


\begin{tabular}{|c|c|c|c|c|c|}
\hline Año & $\begin{array}{l}\text { Denominación } \\
\text { Línea Crediticia }\end{array}$ & $\begin{array}{c}\text { Aporte } \\
\text { Externo } \\
\text { (mills. u\$s) }\end{array}$ & $\begin{array}{c}\text { Contraparte } \\
\text { Local } \\
\text { (mills. u\$s) }\end{array}$ & $\begin{array}{c}\text { Total } \\
\text { (mills. u\$s) }\end{array}$ & $\begin{array}{l}\text { Institución } \\
\text { beneficiaria }\end{array}$ \\
\hline 1979 & BID I & 66 & 69 & 135 & CONICET \\
\hline 1986 & BID II & 61 & 90 & 151 & CONICET \\
\hline 1993 & PMT-I & 95 & 95 & 190 & $\begin{array}{l}\text { CONICET } \\
\text { SECYT } \\
\text { ANPCYT * }\end{array}$ \\
\hline 1999 & PMT-II & 140 & 140 & 280 & ANPCYT \\
\hline 2006 & PMT-III & 280 & 230 & 510 & ANPCYT \\
\hline 2009 & PIT-I & 100 & 27 & 127 & ANPCYT \\
\hline 2011 & PIT-II & 200 & 68 & 268 & ANPCYT \\
\hline 2012 & PIT-III & 200 & 66 & 266 & ANPCYT \\
\hline 2015 & PIT-IV & 150 & 40 & 190 & ANPCYT \\
\hline \multicolumn{2}{|c|}{ Total } & 1292 & 825 & 2117 & \\
\hline \multicolumn{2}{|c|}{ Porcentajes } & $61 \%$ & $39 \%$ & $100 \%$ & \\
\hline
\end{tabular}

Cuadro 1: Créditos externos acordados con el BID para financiar la CTI Fuente: Elaboración propia en base a documentos oficiales del BID. * A partir de su creación en 1996

plicó un retorno parcial a políticas sectoriales que habían caracterizado al sector previo a los 90's, por un lado, ejecutadas desde la modalidad de fondos tecnológicos (por ejemplo a través del Fondo Argentino Sectorial -FONARSEC-) y, por el otro, a través de las instituciones creadas en el marco de la estrategia ISI, como el Consejo Nacional de Investigaciones Científicas y Técnicas (CONICET), el INTA, el INTI y la CNEA, las cuales tuvieron aumentos significativos en sus partidas presupuestarias, lo que permitió una recuperación parcial del rol que tenían con anterioridad en términos de planificación del sector.

El BID acompañó activamente este proceso de transformación en las políticas de CTI argentinas. A continuación, describimos primero el peso relativo del apoyo del BID dentro del presupuesto del sector para luego referirnos a cómo se dio dicho acompañamiento y, finalmente, reflexionar sobre la importancia que tuvo a lo largo de este proceso.

\section{El peso del BID en el financiamiento de las políticas de CTI argentinas}

En América Latina, el BID ha sido la principal fuente de financiamiento internacional para proyectos orientados a actividades de CTI, siendo Argentina el país que acaparó la mayor cantidad de recursos del organismo para esta finalidad (Aguiar, Aristimuño \& Magrini, 2015). Sin embargo, la CTI representa menos del $2 \%$ de la cartera de préstamos del BID. A pesar de esto, veremos que este banco jugó un papel muy importante en las transformaciones de la política de CTI de Argentina en el período de análisis.

La relación del Estado argentino y el BID en temas de CTI comenzó a ser importante a partir de 1979, cuando se firmó el primer contrato programa conocido como BID I. Desde entonces, y hasta 2015, se firmaron ocho nuevos contratos. En el cuadro 1 se resumen todas las operaciones que el Estado argentino firmó con el BID en la finalidad CTI. A su vez, 
se indica el monto total de cada préstamo, qué proporción aportó cada uno y cuál fue el organismo estatal que se benefició con cada contrato programa. Lo primero que se observa claramente es que el BID pasó de aportar una parte menor a la del Estado nacional, del financiamiento total, en los programas BID I y BID II, a financiar primero en partes iguales (Programa de Modernización Tecnológica -PMT- I y II), y finalmente, a aportar la parte mayoritaria del financiamiento a partir del PMT III. Esto fue muy marcado en los Programas de Innovación Tecnológica (PIT) que comenzaron en 2009, donde la proporción fue, a grandes rasgos, de 3 a 1. Y lo segundo es que sólo dos instituciones fueron beneficiarias del apoyo del BID: el CONICET, hasta 1996, y la ANPCyT a partir del mismo año, momento en el que esta última institución fue creada y el PMT-I es reformulado, pasando a ser la ANPCyT el socio ejecutor de los programas acordados con el BID, relación que permanece hasta la actualidad.

Los préstamos del BID fueron importantes para financiar las actividades de la ANPCyT. Según muestra Angelelli (2011), desde su creación hasta 2003 , aproximadamente un $30 \%$ de los recursos de la ANPCYT eran proveídos por esa fuente externa. Sin embargo, a partir de ese año, la relación se invirtió y desde entonces, el BID pasó a representar entre el 60 y el $70 \%$ de los recursos de la agencia. Sin embargo, esto no debe llevar a sobrestimar el peso del BID como financista del sector de CyT argentino. A lo largo del período (1996-2010), los fondos ejecutados por la ANPCYT representaron entre un 5 y un 15\% del presupuesto total de la finalidad Ciencia y Técnica del presupuesto nacional. Sólo para tener una referencia, el CONICET, que se financia íntegramente con recursos del Estado, ejecutó a lo largo del período entre el 25 y el $40 \%$ de los recursos totales del sector (Aristimuño, 2018).

\section{¿Qué políticas de CTI financió el BID entre 1990 y $2015 ?$}

Como se mencionó antes, los primeros créditos del BID a la Argentina para el sector de CTI se remontan a 1979 y 1986, ambos asignados al CONICET. El primero, para financiar el desarrollo de centros regionales y promover investigaciones aplicadas en áreas estratégicas (Algañaraz \& Bekerman, 2014), en el marco de una política de descentralización del sistema científico y tecnológico al mismo tiempo que el sistema universitario enfrentó un proceso de profunda contracción (Bekerman, 2009). Y el segundo, para financiar instrumentos de promoción de la ciencia aplicada, proyectos de investigación y desarrollo (PID), y modernización del equipamiento de laboratorio, tanto en universidades como en Institutos del CONICET. En esta etapa, el BID se orientó a apoyar el fortalecimiento de las capacidades de I+D a través de inversiones en infraestructura y equipamiento, y fondos para becas y proyectos científicos de transferencia potencial al sector privado. El objetivo era elevar la "oferta" de conocimientos, lo cual, se creía, tendría un correlato en la adopción y generación de nuevas tecnologías (Aguiar, Aristimuño \& Magrini, 2015).

A lo largo de la década del '80, el BID actualizó su política de CTI y pasó a reconocer como central la necesidad de implementar políticas de financiamiento a la demanda. En este sentido, se consideraba que el Estado debía actuar como facilitador del desarrollo mediante la resolución de fallas de mercado sin afectar las ventajas comparativas de cada país (Lavarello \& Sarabia, 2015; Mayorga, 1997). En este marco, el BID estableció como línea estratégica el otorgamiento de créditos a unidades descentralizadas para implementar programas de desarrollo tecnológico orientados a promover la innovación en las empresas privadas, así como tam- 
bién la vinculación entre éstas y las instituciones del sistema público de investigación (Aristimuño, Aguiar, \& Magrini, 2017). Esto es, instrumentos de promoción a la innovación y el cambio tecnológico de carácter horizontal.

En 1993, la Secretaría de Programación Económica (SPE) comenzó negociaciones con el BID para una operatoria destinada íntegramente a la modernización tecnológica. En la formulación original, el PMT I se dividía en dos subprogramas ${ }^{3}$ : uno que representaba una novedad institucional, el Fondo Tecnológico Argentino (FONTAR), bajo la órbita de la SPE, que administraba créditos a tasa subsidiada (siempre positiva) y de recupero contingente (en caso de éxito tecnológico), pero no subvenciones. El otro, a cargo del consorcio SECYT/CONICET, mediante subvenciones a Proyectos de Vinculación Tecnológica (PVTs), presentados por UVTs asociadas a empresas, para que pudieran desarrollar el proyecto y, si era exitoso, las empresas se comprometían a adquirir o licitar los resultados de la investigación. Estas condiciones generaron una clara asimetría con los créditos que otorgaba el FONTAR con el mismo fin y con tasa de interés positiva (Del Bello, 2014). Además, en el marco de este subprograma el CONICET recibió parte de los fondos para continuar financiando los PID que ya habían sido financiados con el BID $\|^{4}$.

En 1996, en el marco de la denominada segunda reforma del Estado, se produce una profunda reforma institucional del sistema de CTI, lo que dará lugar a la creación de la ANPCyT a través del Decreto $N^{\circ} 1.660$. A través de dicha reforma se buscó incrementar la eficiencia del sistema a través de la diferenciación institucional de las funciones de formulación de políticas, financiamiento y ejecución de actividades de CTI (Angelelli, 2011; Del Bello, 2014). En este esquema la SECyT quedaría a cargo de la formulación de políticas, ANPCYT a cargo del financiamiento de proyectos de CTI, y el resto de las instituciones del sistema (INTI, INTA, CONEA, Universidades, etc) como ejecutoras de las actividades de CTI. Disponer de un organismo dedicado exclusivamente al financiamiento de las actividades de CTI demandó la reestructuración del PMT I pactado con el BID.

De esta forma, el FONTAR fue transferido a la órbita de la ANPCyT, y se creó un segundo fondo, el Fondo para la Investigación Científica y Tecnológica (FONCyT), integrado con los recursos del PMT I inicialmente asignados al CONICET y a la SECYT. Los PID pasaron a la órbita del FONCyT el cual, además, sumó un nuevo instrumento de promoción a la investigación científica: los Proyectos de investigación científica y tecnológica (PICT). Esta inclusión fue clave para garantizar la aceptación de la ANPCyT por parte de la comunidad científica, ya que, con los PICT, se incrementaron significativamente los recursos disponibles para proyectos de investigación. Los PICT terminaron duplicando el financiamiento de los PID al final del período, posicionándose como el principal instrumento para la investigación científica en el país (Aristimuño \& Aguiar, 2015; Codner, Kirchuk, Aguiar, Benedetti \& Barandiarán, 2006). Esto contribuyó a ganar el apoyo entre los investigadores del CONICET, que originalmente vieron en la creación de la ANPCyT una amenaza a la autonomía de la institución.

\footnotetext{
${ }^{3}$ La división del PMT I en dos subprogramas respondió al hecho de que cuando la operatoria estaba pronta a aprobarse, la SECyT y el CONICET insistieron en que debían ser incorporados. Después de todo, la SECyT era el organismo de aplicación de la Ley 23.877 y a quien correspondía la ejecución de instrumentos de fomento a la CyT. Además, el CONICET había sido históricamente la institución beneficiaria de los programas del BID y aún estaba ejecutando los fondos del BID II (Aguiar, Aristimuño \& Magrini, 2015).

${ }^{4}$ Vale la pena aclarar que, en ese entonces, el secretario de CyT, Raúl Matera, era a su vez presidente del CONICET dado que intervino el organismo en 1991 (Aristimuño \& Aguiar, 2015).
} 
Adicionalmente, tras la reformulación, se eliminaron los instrumentos de crédito de devolución contingente del FONTAR y fueron reemplazados por aportes no reembolsables (ANR). Esto se debe a que, en la práctica, se mostró que los prestatarios se esforzaban por demostrar el fracaso tecnológico para así evitar devolver la totalidad del préstamo. Lo cual llevaba a que, en la práctica, la línea funcione como una subvención, pero con un mayor costo administrativo. Finalmente, la emergencia de nuevas miradas teóricas que asignaban a las pequeñas y medianas empresas (PyMEs) un rol clave en la dinamización del sistema nacional de innovación, motivaron la inclusión de mecanismos pro-activos para garantizar la participación de este segmento empresario en los mecanismos promocionales (Yoguel, Sztulwark y Lugones, 2007).

De esta forma, la ANPCyT se constituyó en la autoridad de aplicación de la Ley 23.877 y, a su vez, en el organismo encargado de la ejecución del PMT I, combinando la promoción de la investigación científica y la innovación empresarial (Lugones, Porta $\&$ Codner, 2013). De acuerdo con Angelelli (2011) y Del Bello (2014), uno de los modelos seguidos para la creación de la ANPCyT fue el de la Financiadora de Estudios y Proyectos (FINEP) de Brasil, en el sentido de diferenciar, por un lado, las funciones de financiamiento y ejecución, y por el otro, las de formulación de políticas científicas y tecnológicas.

El PMT II, acordado en 1999 por la misma gestión que creó el FONTAR y reformuló el PMTI, no implicó grandes cambios respecto al préstamo anterior. De algún modo, este préstamo, el más grande otorgado hasta entonces, fue una forma de dar continuidad a las transformaciones acaecidas a mediados de la década del '90.

A partir de 2003, habiendo pasado la crisis de 2001 y abandonado el patrón de convertibilidad, el país comenzó un período de fuerte crecimiento con superávit comercial y fiscal que sentó las bases para replantear nuevamente la política de CTI. Esto implicó el impulso de ejercicios de planificación tendientes a generar metas y objetivos transversales a mediano y largo plazo, lo que dio lugar, en 2006, a un nuevo Plan de CTI: el Plan del Bicentenario. Por otro lado, ese mismo año, se acordó un tercer tramo del PMT con el BID y se creó el Fondo Fiduciario de Promoción de la Industria del Software (FONSOFT), como resultado de la aplicación de la Ley 25.922 que había sido sancionada en 2004.

Estos tres hechos, según Lavarello \& Sarabia (2015), implicaron el retorno parcial a una visión de planificación estatal sectorial, centrada en el desarrollo de capacidades tecnológicas en sectores con potencialidad de difusión intersectorial de conocimientos. Decimos parcial, porque los instrumentos horizontales, así como el enfoque de subsidio a la demanda, continuaron vigentes. De esta forma, el enfoque sectorial se concibió por un lado, como un modelo de intervención complementario al horizontal, al introducir recursos sectorialmente reservados. Por otro lado, con la creación de los Proyectos de Desarrollo de Aglomerados Productivos (PITEC), pactados con el BID en la nueva operatoria, se formalizaba el objetivo de materializar la idea de cluster científico tecnológico5.

Los PI-TEC del PMT III adelantaron la creación en 2009 del FONARSEC (Fondo Argentino Sectorial). El cual sería financiado en parte por el BID, a través del PIT I, y, por el Banco Internacional de Recons-

\footnotetext{
${ }^{5}$ Los PI-TEC financian grupos empresarios y/o de investigación en áreas científico-tecnológicas prioritarias, concentrados regionalmente y con potencialidad competitiva a escala internacional, mediante los diferentes instrumentos del que disponen el FONCYT y FONTAR. Finalmente, el Plan del Bicentenario señalaba cuáles eran las áreas prioritarias para focalizar los recursos. Sin embargo, como dato curioso que habla de las dificultades para tomar medidas planificadas en un área como la CTI, es que al momento de presentar el Plan del Bicentenario aún no se había creado el FONSOFT y, por lo tanto, este instrumento r no figuraba como una opción para financiar áreas prioritarias.
} 
| Ciencia, Tecnología y Política | Año 2 | No3 | Julio-Diciembre 2019 | ISSN 2618-3188 | www.revistas.unlp.edu.ar/CTyP |

trucción y Fomento (BIRF) del Banco Mundial. Esta sería la primera operación de préstamo del BIRF con Argentina para temas de CTI (Loray, 2018). El FONARSEC, mediante distintos fondos buscó impulsar la asociatividad público-privada y la innovación en sectores estratégicos y en TPG. De esta forma, se introdujeron mecanismos de intervención en concordancia con los nuevos lineamientos estratégicos tendientes a priorizar el desarrollo sectorial y TPG contenidos en el Plan Bicentenario y el Plan Argentina Innovadora 2020. En otros términos, posibilitó la implementación de instrumentos de financiación de carácter selectivo orientados al desarrollo de sectores de alta tecnología de carácter transversal para la resolución de demandas tecnológicas de "núcleos socio-productivos" estratégicos ${ }^{6}$.

De esta forma, la ANPCYT, mediante sucesivos acuerdos con el BID, fue introduciendo cambios en sus modalidades de apoyo que le permitieron constituir una oferta diversificada de instrumentos que combinan modalidades de intervención horizontales y focalizadas, así como también el fortalecimiento de capacidades tecnológicas a través de consorcios público-privados? ${ }^{7}$. Por otro lado, es necesario notar que, con excepción del FONCyT, los distintos fondos que integran dicho organismo están explícitamente dirigidos al sector productivo ${ }^{8}$. Finalmente, el FONTAR y el FONSOFT tienen un claro sesgo hacia el segmento de firmas PyMEs mediante criterios selectivos en la asignación de los recursos (ver tabla †).

\begin{tabular}{|l|l|l|}
\hline Fondo & Modalidad & Beneficiario \\
\hline FONCVT & Subsidios & $\begin{array}{l}\text { Grupos de I+D de } \\
\text { instituciones de CTI públicas } \\
\text { o privadas sin fines de lucro }\end{array}$ \\
\cline { 1 - 2 } FONTAR & $\begin{array}{l}\text { Incentivos } \\
\text { fiscales }\end{array}$ & Empresas \\
\cline { 1 - 2 } FONTAR & $\begin{array}{l}\text { Subsidios } \\
\text { Créditos }\end{array}$ & $\begin{array}{l}\text { Empresas } \\
\text { Eentros Tecnológicos } \\
\text { EOnsrendedores } \\
\text { Consorcios públicos-privados }\end{array}$ \\
\cline { 1 - 1 } FONARSEC & & \\
\hline
\end{tabular}

Tabla 1: Tipología de los instrumentos de la ANPCYT según tipo de Fondo. Fuente: elaboración propia en base a datos del ANPCyT.

Adicionalmente, al analizar las características de las líneas crediticias es posible apreciar las condiciones impuestas por el BID para otorgar su apoyo financiero. Especialmente en el caso del FONTAR, donde los créditos fondeados con recursos del organismo internacional requieren de la participación de intermediarios financieros, bancos comerciales tanto públicos como privados, mientras que los créditos otorgados con recursos del tesoro nacional son otorgados en forma directa. En el primer caso el FONTAR opera como banco de segundo piso, limitando su actuación a evaluar el sustento tecnológico de los proyectos, mientras que la banca comercial evalúa la sustentabilidad financiera y el riesgo del cliente ${ }^{9}$. Por el contrario, las líneas de crédito fondeadas a través de recursos públicos asignados en el marco de la aplicación de la Ley 23.877, son operadas por el

\footnotetext{
${ }^{6}$ El Plan Argentina Innovadora 2020, presentado en 2012, identifica 34 núcleos socio-productivos correspondiente a los siguientes sectores considerados estratégicos: agroindustria, ambiente y desarrollo sustentable, desarrollo social, energía, industria y salud. Mientras que los denominados sectores de alta tecnología son TIC, biotecnología y nanotecnología.

${ }^{7}$ Según Barletta, Moori Koenig \& Yoguel (2014), con relación a las modalidades e instrumentos dirigidos a atender las demanda del sector productivo, es posible observar respecto del FONTAR, que entre 2006 y 2012, el principal instrumento fueron las distintas líneas crediticias (44\%), seguido de los subsidios directos (34\%), los incentivos fiscales (16\%) y, finalmente, los instrumentos de carácter asociativo (6\%). ${ }^{8}$ Lo cual se aprecia en el hecho de que el sector productivo recibió el 79\% de los recursos asignados entre 2009 y 2015, siendo el FONTAR el instrumento más importante, habiendo adjudicado el 46\% de los recursos totales de dicho período (Barletta, Moori Koenig \& Yoguel, 2014). Una explicación acabada de esta dinámica requiere considerar las características de cada instrumento en términos de condiciones de acceso y monto por proyecto, así como también la secuencia temporal entre convocatoria, evaluación y adjudicación de proyectos.

${ }^{9}$ Este es un aspecto clave que permite diferenciar a la ANPCyT de otros organismos de la región, como es el caso de la FINEP de Brasil (Aristimuño \& Aguiar, 2015; Del Bello, 2014; Peirano, 2011).
} 
FONTAR en calidad de banco de primer piso. Esto permite flexibilizar los requisitos de acceso y las condiciones financieras facilitando la participación de las empresas de menor tamaño relativo (Barletta, Moori Koenig \& Yoguel, 2014). Que el FONTAR funcione como banco de segundo piso en el caso de recursos prestados por el BID fue una de las condiciones que el organismo impuso a los funcionarios argentinos (Aguiar, Aristimuño, \& Magrini, 2015).

\section{Conclusiones}

En este trabajo se analizaron 25 años de relación entre el BID y las políticas de CTI en Argentina. Lo primero que se debe destacar es que el BID cumplió un rol significativo en la consolidación de la ANPCyT, considerando el peso que el mismo tiene sobre el total de recursos de los que dispuso dicho organismo, peso que se fue incrementando en los sucesivos acuerdos realizados en la renovación del PMT y el PIT (ver CUADRO 1). Además, cada nuevo préstamo del BID implicó un encadenamiento con los anteriores. Es necesario aclarar, sin embargo, que como ya se dijo, la ANPCyT sólo da cuenta de una pequeña porción de los recursos del sector, dependiendo del año, entre un 5 y un $15 \%$ de los recursos de la finalidad CTI en el presupuesto.

El apoyo del BID permitió dar continuidad a una serie de transformaciones que comenzaron con la sanción en 1990 de la Ley 23.877 y que dio lugar a la implementación de un nuevo paradigma de política de CTI basado en la promoción de la innovación empresarial a través del subsidio a la demanda. En otras palabras, el BID, al garantizar una masa estable de recursos, otorgó a la ANPCyT la consistencia presupuestaria que le permitió consolidar a lo largo del tiempo este nuevo paradigma de políticas.
La consolidación de la ANPCyT como uno de los actores centrales del sistema de CTI, tuvo ciertos efectos que se pueden apreciar en: 1- la implementación de un nuevo marco normativo que permite otorgar beneficios económicos (tanto de índole financiera como no financiera) de forma directa a las empresas. 2- la introducción de nuevos mecanismos de asignación de recursos a través del sistema de concursos públicos y de estándares internacionales de evaluación de la pertinencia y calidad de los proyectos tanto científicos como tecnológicos. Y 3- un proceso de acumulación de capacidades institucionales que se tradujo en la ampliación de la oferta de instrumentos de promoción y la complementariedad entre instrumentos horizontales y selectivos.

En consecuencia, el BID fue una condición de posibilidad en la implementación de esta nueva modalidad de intervención estatal en el campo de la CTI. Es importante destacar, a la luz de los cambios operados sobre la misma, que el BID mostró una trayectoria de aprendizaje que llevó a que modificara sus recomendaciones e instrumentos financiados a lo largo del tiempo. El estudio de la evolución de los marcos conceptuales que fueron utilizados en los distintos períodos, así como la forma en que son socialmente construidos, sigue siendo una de las llaves para comprender las transformaciones en las políticas de los países donde el banco participa.

No obstante estas observaciones, es posible advertir que este nuevo enfoque de intervención presentó ciertos límites. En primer lugar, no tuvo efectos significativos en lo que refiere a una mayor coordinación interinstitucional. Es decir, no se modificó el perfil institucional heterogéneo y aislado del complejo de CTI argentino, subsistiendo problemas de gobernanza y de superposición entre las actividades y misiones de las distintas 
instituciones.

En segundo lugar, la evidencia disponible da cuenta de que el acceso a los beneficios promocionales de subsidio a la demanda reposa en la iniciativa y capacidad de los actores. Lo cual presenta el riesgo de profundizar las asimetrías pre-existentes en la estructura productiva del país y financiar recurrentemente las actividades de un mismo grupo de empresas con capacidad para la captura de estos fondos públicos. Habiendo transcurrido más de dos décadas en la aplicación de estas políticas de subsidio horizontal a la demanda, no se pueden observar grandes resultados en cuanto al objetivo que inicialmente llevó a su adopción, esto es, impulsar una mayor participación del sector privado en el financiamiento de la CTI. Finalmente, en este mismo sentido, una debilidad importante de la ANPCyT, pero también del resto de las instituciones del complejo CTI argentino, es la ausencia de un sistema público y detallado de datos y estadísticas y que permita poder analizar con mayor precisión los impactos de las políticas que se aplican.

En tercer y último lugar, si bien el BID mostró flexibilidad para la introducción de cambios en la operatoria de la ANPCyT, por ejemplo, al permitir la implementación de subsidios para empresas, esto no implicó que no haya impuesto límites en el diseño de los instrumentos. Por ejemplo, estableció como condición que la ANPCyT no puede actuar como banco de primer piso para otorgar créditos a tasa subsidiada, algo que los negociadores argentinos proponían .

\section{Bibliografía}

Aguiar, D., Aristimuño, F., \& Magrini, N. (2015). El rol del Banco Interamericano de Desarrollo (BID) en la reconfiguración de las instituciones y políticas de fomento a la ciencia, la tecnología y la innovación de la Argentina (1993-1999). REVISTA CTS, 10(29), 11-40. Recuperado de https://dialnet.unirioja.es/ servlet/articulo?codigo $=5130672$

Algañaraz, V., \& Bekerman, F. (2014). El préstamo BID-CONICET: un caso de dependencia financiera en la política científica de la dictadura militar argentina (1976-1983). En F. Beigel \& H. Sabea (Eds.), Dependencia académica y profesionalización en el Sur: perspectivas desde la periferia (pp. 129-139). Mendoza: EDIUNC.

Angelelli, P. (2011). Características y evolución de la Agencia Nacional de Promoción Científica y Tecnológica. En F. Porta \& G. Lugones (Eds.), Investigación científica e innovación tecnológica en Argentina. Impacto de los fondos de la Agencia Nacional de Promoción Científica y Tecnológica. (pp. 67-79). Bueno Aires: Universidad Nacional de Quilmes.

Anlló, G., \& Peirano, F. (2005). Una mirada de los sistemas nacionales de innovación en el MERCOSUR: análisis y reflexiones a partir de los casos de Argentina y Uruguay (Estudios y perspectivas) (Vol. 22). Buenos Aires: United Nations Publications. Recuperado de https://repositorio.cepal.org/hand le/11362/4849

Aristimuño, F. (2018). Construcción de las políticas de ciencia y tecnología en la Secretaría de Ciencia y Tecnología de Argentina (1989-1999). Un análisis desde la perspectiva de las culturas políticas. Universidad Nacional de Rio Negro. Recuperado de https://rid.unrn.edu.ar/jspui/hand le/20.500.12049/1233

Aristimuño, F. (2019). De Institutos a Fondos Tecnológicos : la transformación del Estado argentino en la década de 1990. Realidad Económica, (323), 9 a 36. Recuperado de http://www.iade.org.ar/arti culos/de-institutos-fondos-tecnologicos-la-trans formacion-del-estado-argentino-en-la-decada-de Aristimuño, F., \& Aguiar, D. (2015). Construcción de 
las políticas de ciencia y tecnología en Argentina desde 1989 a 1999. Un análisis de la concepción de las políticas estatales. REDES. Revista de Estudios Sociales de la Ciencia, 21(40). Recuperado de https://ridaa.unq.edu.ar/handle/20.500.11807/367

Aristimuño, F., Aguiar, D., \& Magrini, N. (2017). ¿Transferencia de modelos institucionales o redes de asuntos de expertos? Análisis de un préstamo para ciencia y tecnología en Argentina del Banco Interamericano de Desarrollo durante los noventa. Estudios Sociales del Estado, 3(5), 99-131. Recuperado de http://www.estudiossocialesdelestado. org/index.php/ese/article/view/108

Barletta, F., Moori Koening, V. y Yoguel, G. (2014): "Políticas e instrumentos para impulsar la innovación en las pymes argentinas", en M. Dini, S. Rovira. y G. Stumpo, G. (comps.): Una promesa y un suspirar. Políticas de innovación para pymes en América Latina, Santiago de Chile, CEPAL/GIZ/BMZ, pp. 2369. Recuperado en: https://repositorio.cepal.org/ handle/11362/39719

Bekerman, F. (2009). El campo científico argentino en los años de plomo: desplazamientos y orientación de los recursos. Sociohistórica, (26), 151-166.

Codner, D., Kirchuk, E., Aguiar, D., Benedetti, G., \& Barandiarán, S. (2006). Evaluación de instrumentos de promoción científica y tecnológica: el caso del Proyecto de Investigación Científica y Tecnológica (PICT) en Argentina. Redes, 12(24), 131-150. Recuperado de https://ridaa.unq.edu.ar/hand le/20.500.11807/619

Del Bello, J. C. (2014). Argentina: Experiencia de transformación de la institucionalidad pública de apoyo a la innovación y al desarrollo tecnológico. En J. C. Del Bello, G. Rivas, \& S. Rovira (Eds.), América Latina: Experiencia de transformación de la institucionalidad pública de apoyo a la innovación y al desarrollo tecnológico (pp. 35-78). Recuperado de https://repositorio.cepal.org/handle/11362/37083 Hurtado, D., Lugones, M., \& Surtayeva, S. (2017). Tecnologías de propósito general y políticas tecnológicas en la semiperiferia- El caso de la nanotecnología en la Argentina. Revista CTS, 12(34), 65-93. Recuperado de https://dialnet.unirioja.es/servlet/ articulo?codigo $=6060822$

Lavarello, P. y Sarabia, M. (2015). La política industrial en la Argentina durante la década de 2000 (Estudios y Perspectivas). Buenos Aires. Recuperado de https://repositorio.cepal.org/handle/11362/39886

Loray, R. P. (2018). Organismos internacionales y políticas de ciencia, tecnología e innovación. El rol del Banco Interamericano de Desarrollo en el diseño e implementación de los Fondos de Innovación Tecnológica Sectorial de Argentina (2009-2015). Universidad Nacional de Quilmes. Recuperado de https://ridaa.unq.edu.ar/hand le/20.500.11807/819?show=full

Lugones, G., Porta, F., \& Codner, D. (2013). Perspectiva sobre el impacto del Programa de Modernización Tecnológica del BID en la política de CTI de Argentina. En Políticas de ciencia, tecnología e innovación para el desarrollo. La experiencia latinoamericana (pp. 69-91). México D.F: FCCyT/LALICS.

Mayorga, R. (1997). Cerrando la brecha. Washington DC.

Peirano, F. (2011). El FONTAR y la promoción de la innovación en empresas entre 2006 y 2010. En F. Porta \& G. Lugones (Eds.), Investigación científica e innovación tecnológica en Argentina. Impacto de los fondos de la Agencia Nacional de Promoción Científica y Tecnológica. (pp. 81-131). Universidad Nacional de Quilmes.

Sztulwark, S. (2010). Políticas e instituciones de apoyo a las pymes en la Argentina. En C. Ferraro \& G. Stumpo (Eds.), Políticas de apoyo a las pymes en América Latina. Entre avances inno- 
vadores y desafíos institucionales (pp. 45-96).

Disponible en: https://repositorio.cepal.org/hand le/11362/2555

Teubal, M. (1990): "Lineamientos para una política de desarrollo industrial y tecnológica: la aplicabilidad del concepto de las distorsiones del mercado", en Ffrench-Davis, R.; Teubal, M. y Ros, J.: Elementos para el diseño de políticas industriales y tecnológicas en América Latina, Cuadernos de la CEPAL N 63, Santiago de Chile, CEPAL, pp. 65118. Disponible en: https://repositorio.cepal.org/ handle/11362/2714

Yoguel, G., Lugones, M. y Sztulwark, S. (2007): La política científica y tecnológica Argentina en las últimas décadas: algunas consideraciones desde la perspectiva del desarrollo de procesos de aprendizaje, Manual de Políticas Públicas, Santiago de Chile, CEPAL-GTZ. 